\title{
Quantum Randomness Certified by the Uncertainty Principle
}

\author{
Giuseppe Vallone, Davide G. Marangon, Marco Tomasin, and Paolo Villoresi \\ Department of Information Engineering, University of Padova, I-35131 Padova, Italy
}

\begin{abstract}
We present an efficient method to extract the amount of true randomness that can be obtained by a Quantum Random Number Generator (QRNG). By repeating the measurements of a quantum system and by swapping between two mutually unbiased bases, a lower bound of the achievable true randomness can be evaluated. The bound is obtained thanks to the uncertainty principle of complementary measurements applied to min- and max- entropies. We tested our method with two different QRNGs, using a train of qubits or ququart, demonstrating the scalability toward practical applications.
\end{abstract}

\section{INTRODUCTION}

Random numbers are of fundamental importance for scientific and practical applications. On the last years, great effort has been devoted to Quantum Random Number Generators (QRNG), based on the intrinsic randomness of the quantum measurement process $[1-10$. Theoretical analyses about the security and the real content of randomness have been given only recently $[9-13$. It has been shown that true random numbers, namely uniform and uncorrelated from any classical or quantum sideinformation held by an eavesdropper, can be achieved by using the randomness expansion [13, 14] or amplification protocols [7, 15. Expansion refers to a protocol able to generate true random numbers by starting with a short random seed. In an amplification protocol, the initial seed can have arbitrarily weak (but nonzero) randomness at the price of lower output rate. However, both protocols are very demanding under the experimental point of view since, by operating in the device independent framework, the only way to get perfect randomness is to enforce conditions of no-locality and no-signalling between two parties that violate a (loophole-free) Bell inequality [9].

A general QRNG works as follows: given a $d$-level quantum system $A$ prepared in a state $\rho_{A}$, the random variable $Z$ is obtained by measuring the state $\rho_{A}$ with a $d$ outcome measurement $\mathbb{Z}$ : each outcome $z$ is obtained with a given probability $P_{z}$. If the state $\rho_{A}$ is pure, the number of true random bits that can be extracted from each measurement is quantified by the classical minentropy $H_{\infty}(Z)=-\max _{z}\left(\log _{2} P_{z}\right)$. In this work we aim to deal with a generic scenario, in which the state $\rho_{A}$ is not pure and therefore the system $A$ is correlated with another quantum system, denoted by $E$. In this case it is necessary to estimate the amount of (quantum) information that an adversary Eve holding the system $E$ has on the variable $Z$. The importance of this estimation can be illustrated by a simple example. Let's suppose that Eve holds two entangled photons in the state $|\Phi\rangle=\frac{1}{\sqrt{2}}(|H H\rangle+|V V\rangle)$ and sends to Alice one of the two photons as the system she uses for the randomness extraction. If Alice measures in the $\{|H\rangle,|V\rangle\}$ basis she obtains a perfect random bit from the point of view of the classical min-entropy, since the two outcomes, $|H\rangle$ and $|V\rangle$, are equally probable. However, due to the correlations in the $|\Phi\rangle$ state, Eve knows perfectly the outputs of Alice's measurements: the "random" bit held by Alice can be predicted with certainty by Eve.

The amount of true random bits that can be extracted from the random variable $Z$, if one requires uniformity and independence from the environment system $E$, is given by the conditional min-entropy $H_{\min }(Z \mid E)[16,17$. Indeed, the probability of guessing $Z$ by holding the quantum system $E$ is given by [18]

$$
p_{\text {guess }}(Z \mid E)=2^{-H_{\min }(Z \mid E)} .
$$

For instance, in the previous example with the entangled state $|\Phi\rangle, p_{\text {guess }}(Z \mid E)=1$ and the system held by Alice doesn't allow the generation of true random numbers.

We will present a method, based on the Uncertainty Principle (UP), to estimate the conditional min-entropy and then the amount of true randomness that can be obtained by a given source. We will show and experimentally test that, by measuring the system in conjugate observables $\mathbb{Z}$ and $\mathbb{X}$, it is possible to obtain the following bound on the conditional min-entropy

$$
H_{\min }(Z \mid E) \geq \log _{2} d-H_{1 / 2}(X)
$$

where $d$ is the dimension of the Hilbert space and $H_{1 / 2}(X)$ the max-entropy of $\mathbb{X}$ outcomes (see below). The measurement $\mathbb{Z}$ is used to generate the random sequence $Z$, while the measurement $\mathbb{X}$ is used to quantify the amount of true-randomness contained in $Z$. In our protocol we do not use any assumption on the source $\rho_{A}$ : an adversary, called Eve can have full control on the source and the environment $E$. The bound 2 is achieved by only assuming trusted measurements device, meaning that Eve has no access to it and that the device performs a given POVM that are only sensitive to a subspace of dimension $d$. To prevent the possibility that an adversary controls the detection efficiency, as reported in quantum hacking against detectors [19 21, it is necessary to monitor all detector parameters, such as bias voltage, current, and temperature 22. The advantage of the presented method resides on its simplicity: no Bell inequality violation is required but it is only necessary to measure the system in two conjugate bases. With an initial seed of true randomness, our protocol is able to ex- 
pand the randomness by taking into account all possible side quantum information possessed by Eve.

\section{PROOF OF MAIN RESULT}

In this section we derive our main result (2). We first start by reviewing the uncertainty relation for min- and max- conditional entropies introduced in $[23,25]$.

\section{A. Uncertainty principle}

Let's consider three quantum systems $A, B$ and $E$ and $\rho_{A B E}$ a tripartite state. Define $\mathbb{Z}$ and $\mathbb{X}$ as two POVMs on $A$ with elements $\left\{\hat{\mathcal{M}}_{z}\right\}$ and $\left\{\hat{\mathcal{N}}_{x}\right\}$, and random outcomes $Z$ and $X$ encoded in two orthonormal bases $\{|z\rangle\}$ and $\{|x\rangle\}$. Then, the uncertainty principle is written as

$$
H_{\min }(Z \mid E)_{\rho}+H_{\max }(X \mid B)_{\rho} \geq q,
$$

where the min-entropy and max-entropy (see Appendix A1 and [18, for min- and max- entropy definition) are evaluated on the post-measurement states $\rho_{Z E} \equiv \sum_{z}|z\rangle\left\langle z\left|\otimes \operatorname{Tr}_{A B}\left[\hat{\mathcal{M}}_{z} \rho_{A B E}\right], \rho_{X B} \equiv \sum_{x}\right| x\right\rangle\langle x| \otimes$ $\operatorname{Tr}_{A E}\left[\hat{\mathcal{N}}_{x} \rho_{A B E}\right]$ and

$$
q \equiv \log _{2} \frac{1}{c}, \quad c \equiv \max _{z, x}\left\|\sqrt{\hat{\mathcal{M}}_{z}} \sqrt{\hat{\mathcal{N}}_{x}}\right\|_{\infty}^{2} .
$$

The parameter $c$ represents the maximum "overlap" between the two POVMs and $q$ quantifies the "incompatibility" of the measurements. If $\hat{\mathcal{M}}_{z}$ and $\hat{\mathcal{N}}_{x}$ are projective measurements corresponding to Mutually-Unbiased bases in dimension $d$, then $c=\frac{1}{d}$.

\section{B. Proof of the bound}

In a QRNG, Alice measures its system $\rho_{A}$ by using a POVM measurement $\left.\mathbb{Z} \equiv\left\{\hat{\mathcal{M}}_{z}\right\}\right\}^{1}$ The state $\rho_{A}$ is in general correlated with an external system $E$ such that $\rho_{A}=\operatorname{Tr}_{E}\left[\rho_{A E}\right]$. The possible outcomes of the POVM can be encoded in an orthonormal basis $\left\{|z\rangle_{A}\right\}$, such that the post-measurement state is $\rho_{Z E} \equiv \sum_{z}|z\rangle\langle z| \otimes$ $\operatorname{Tr}_{A}\left[\hat{\mathcal{M}}_{z} \rho_{A E}\right]=\sum_{z} P_{z}|z\rangle\langle z| \otimes \rho_{E}^{z}$ with normalized $\rho_{E}^{z}$. Eve's knowledge about the possible outcomes of the $\mathbb{Z}$ measurements is given by the min-entropy $H_{\min }(Z \mid E)$, evaluated over $\rho_{Z E}$. If Alice sometimes measures her system with a different POVM $\mathbb{X}$, the UP allows to bound the min-entropy $H_{\min }(Z \mid E)$ and then the guessing probability by eq. (1). In fact, by using eq. (3) and by considering the system $B$ as a trivial space, the uncertainty relation becomes $H_{\min }(Z \mid E) \geq q-H_{\max }(X)$, where the maxentropy must be evaluated on the state obtained by the

\footnotetext{
1 We employed POVMs to present our method in a general framework, but projective measurements are more suited for practical applications.
}

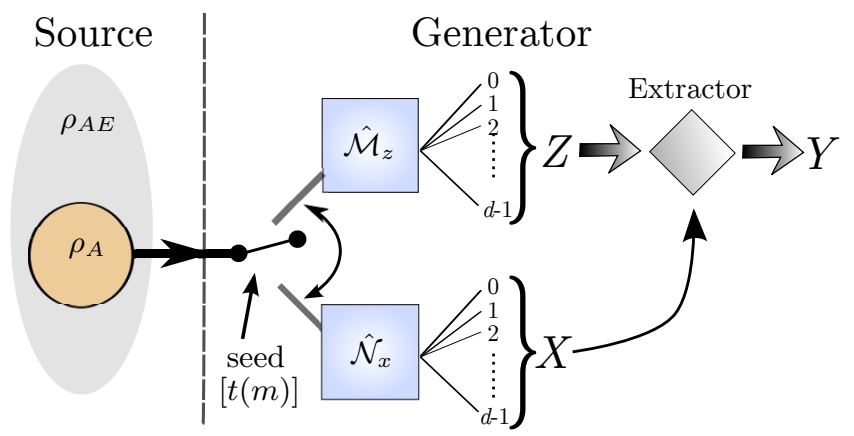

FIG. 1. (Color online) Scheme of the QRNG. The source of randomness is the state $\rho_{A}$ that can be correlated with a larger system $E$. An initial perfect random seed of length $t(m)$ is used to switch between the $\left\{\hat{\mathcal{M}}_{z}\right\}$ and $\left\{\hat{\mathcal{N}}_{x}\right\}$ POVMs, from which the random variables $Z$ and $X$ are extracted. The variable $Z$ is used to generate the random sequence, while the variable $X$ is used to evaluate how many true random bits can be extracted by $Z$. $Y$ represents the final true random sequence.

$\mathbb{X}$ measurement, namely $\rho_{X} \equiv \sum_{x} p_{x}|x\rangle\langle x|$, with $p_{x}=$ $\operatorname{Tr}_{A E}\left[\hat{\mathcal{N}}_{x} \rho_{A E}\right]$. In this case $H_{\max }(X)=2 \log _{2} \operatorname{Tr}\left[\sqrt{\rho_{X}}\right]$ (see Appendix A1 and [18]), i.e. the max-entropy is equal to $H_{1 / 2}(X)$, the Rényi entropy ${ }^{2}$ of order $1 / 2$ of the classical outcome $X$.

Our result can be summarized as follows: the conditional min-entropy of the $\mathbb{Z}$ outputs can be bounded by using the Rényi entropy of order $1 / 2$ of the $\mathbb{X}$ outputs, namely

$$
H_{\min }(Z \mid E) \geq q-H_{1 / 2}(X) .
$$

that reduces to 2 in case of conjugate observables in $d$ dimensions. We would like to point out that, thanks to the inequality $H_{1 / 2}(X)+H_{\infty}(Z) \geq q$ derived by Maassen and Uffink [26], the bound $q-H_{1 / 2}(X)$ is always lower than the classical min-entropy $H_{\infty}(Z)$ evaluated on the probabilities $P_{z}$.

\section{UP-CERTIFIED QRNG}

Let's now evaluate the bound in two particular cases. Let's consider the $\mathbb{Z}$ POVM as projective measurements in the computational basis, $\{|0\rangle,|1\rangle, \cdots,|d-1\rangle\}$ and the $\mathbb{X}$ measurement chosen as its discrete-Fourier transform $|x\rangle=\frac{1}{\sqrt{d}} \sum_{z=0}^{d-1} e^{i \frac{x z}{2 \pi d}}|z\rangle$ for which $q=\log _{2} d$. If the system $A$ is prepared in the state $|\psi\rangle_{A}=\frac{1}{\sqrt{d}} \sum_{z}|z\rangle$, then $H_{1 / 2}(X)=0$ and $(5)$ bounds $H_{\min }(Z \mid E)$ to the classical min-entropy $H_{\infty}(Z)=\log _{2} d$. The random variable $Z$

\footnotetext{
${ }^{2}$ We recall that the Rényi entropy of order $\alpha$ is defined as $H_{\alpha}(X)=\frac{1}{1-\alpha} \log _{2} \sum_{x=0}^{d-1} p_{x}^{\alpha}$.
} 
is then uniformly distributed and independent from any adversary. However, in practical implementations of a QRNG, it is impossible to prepare the system $A$ in a perfect pure state $|\psi\rangle_{A}$. When the state $\rho_{A}$ is not pure, the entropies $H_{\infty}(Z)$ and $H_{\min }(Z \mid E)$ can be different. Our result is thus particularly effective with real sources (that cannot generate pure states) since it bounds the effective achievable randomness without requiring any assumption on them. Even if Eve has complete control on the source $\rho_{A}$, the bound given in (5) evaluates the amount of true random bits that can extracted from $Z$. This randomness has complete quantum origin and no side information can be used to predict the generated random bits.

Another important example is represented by the system described in the introduction: Eve sends to Alice one photon of a two-photon maximally entangled state, and thus can perfectly predict the outputs of Alice's measurements. In this case, Alice holds a completely mixed state $\rho_{A}=\frac{1}{2} \mathbb{1}_{2}$ and the max-entropy is $H_{1 / 2}(X)=1$. Thanks to eq. (5) and (1), the bound on the min-entropy becomes trivial, $H_{\min }(Z \mid E) \geq 0$ and $p_{\text {guess }}(Z \mid E) \leq 1$ : our result correctly predicts that the guessing probability can reach unity and so no true random bits can be extracted in this case.

In order to exploit the result of eq. (5) it is necessary to estimate the max-entropy of the source $\rho_{A}=\operatorname{Tr}_{E}\left[\rho_{A E}\right]$. However, since the POVM $\left\{\hat{\mathcal{M}}_{z}\right\}$ and $\left\{\hat{\mathcal{N}}_{x}\right\}$ are incompatible, it is not possible to measure them at the same time. We then need to switch randomly between $\hat{\mathcal{M}}_{z}$ and $\hat{\mathcal{N}}_{x}$ during the random bit generation (see Figure 1). The measurements are chosen by using a seed of true randomness that our method is able to expand. From this point of view, our method can be seen as a random number expansion protocol.

We now show that the number of random extracted bits is greater than the required seed. Let $m$ the total number of measurements. We decide that, over $m$, the number of measurements in the POVM $\left\{\hat{\mathcal{N}}_{x}\right\}$ will be $n_{X}=\lceil\sqrt{m}$, such that the probability of measuring in the $\mathbb{X}$ basis is approximately $\frac{1}{\sqrt{m}}$. To randomly choose $n_{X}$ among $m$ measurements we need a number of bits given by $t(m)=\left\lceil\log _{2} \frac{m !}{n_{X} !\left(m-n_{X}\right) !}\right\rceil$. This is the length of the random seed required for the randomness expansion.

The probabilities of outcomes in the $\mathbb{X}$ basis are given by $p_{x}=\operatorname{Tr}_{A}\left[\hat{\mathcal{N}}_{x} \rho_{A}\right]$ and the asymptotic lower bound of the min-entropy is $H_{\min }(Z \mid E) \geq q-H_{1 / 2}(X)$. From the experimental point of view we need to estimate the max-Entropy $H_{1 / 2}(X)$ by using the $n_{X}$ outcomes. If we denote by $n_{x}$ the number of outcomes such that $X=x$, we can estimate the max-entropy by using the Bayesian estimator defined in [27] (with a uniform prior distribution):

$$
\widetilde{H}_{1 / 2}\left(\left\{n_{x}\right\}\right)=2 \log _{2}\left[\frac{\Gamma\left(n_{X}+d\right)}{\Gamma\left(n_{X}+d+\frac{1}{2}\right)} \sum_{x=0}^{d-1} \frac{\Gamma\left(n_{x}+\frac{3}{2}\right)}{\Gamma\left(n_{x}+1\right)}\right]
$$

The Bayesian estimator has a lower variance with respect to the frequentist estimator $\widetilde{H}_{1 / 2}^{f}=2 \log _{2}\left[\sum_{x=0}^{d-1} \sqrt{\frac{n_{x}}{n_{X}}}\right]$. Moreover, for low max-entropies, the frequentist estimator has a negative bias that overestimates the bound on the min-entropy.

Then, given $m$ measurements, the number of extracted random bits are the outputs of the $\mathbb{Z}$ measurement, given by $m-n_{X}$ : due to the bound (5), at least $\left(m-n_{X}\right)\left(q-H_{1 / 2}(X)\right)$ are true random bits. If we subtract the number of bits $t(m)$ required for the seed, we can estimate the random bits generation rate per measurement as

$$
\widetilde{r}\left(\left\{n_{x}\right\}\right)=\frac{b_{\mathrm{sec}}}{m},
$$

where $b_{\text {sec }}$ is the number of generated true random bits :

$$
b_{\mathrm{sec}}=\left(m-n_{X}\right)\left[q-\widetilde{H}_{\max }\left(\left\{n_{x}\right\}\right)\right]-t(m) .
$$

It is worth noticing that, in the infinite size limit $m \rightarrow$ $+\infty$, the seed length is given by $t(m) \sim \sqrt{m} \log _{2} \sqrt{m}$, the estimator $\widetilde{H}_{1 / 2}\left(\left\{n_{x}\right\}\right) \sim H_{1 / 2}(X)$, and the rate approaches the asymptotic limit $\widetilde{r} \longrightarrow r(Z)=q-H_{1 / 2}(X)$. Since the number of extracted random bits are quadratically larger than the initial seed bits, the generator can work in loop: an initial seed is expanded and part of the extracted randomness is fed as a new seed.

\section{EXPERIMENTAL REALIZATION}

We have experimentally tested our method with two different random number generators implemented by photon pairs generated in the $|H V\rangle$ state by spontaneous parametric down conversion. See Appendix B for details about the source. The first generator is a single qubit QRNG, operated by an heralded single photon source: one photon of the pair, measured in the $|H\rangle$ state, is used as trigger, while the second represents the signal. By measuring the signal photon in the $\mathbb{Z}=\{|+\rangle,|-\rangle\}$ and $\mathbb{X}=\{|H\rangle,|V\rangle\}$ bases, we generate the random variables $Z$ and $X$. Here we denote with $| \pm\rangle$ the diagonal polarization states $\frac{1}{\sqrt{2}}(|H\rangle \pm|V\rangle)$. The second generator is a 4-level system (ququart) QRNG, represented by the pair of photons. In this case the $\mathbb{Z}$ and $\mathbb{X}$ bases are respectively given by $\{|++\rangle,|+-\rangle,|-+\rangle,|--\rangle\}$ and $\{|H V\rangle,|V V\rangle,|H H\rangle,|V H\rangle\}$.

We first analyze the qubit QRNG. By choosing different values of $m$ we performed $n_{X}=\lceil\sqrt{m}\rceil$ measurements in the $\mathbb{X}$ basis and $n_{Z}=m-n_{X}$ measurements in the $\mathbb{Z}$ basis, obtaining the sequences $X$ and $Z$. The two sequences are used to estimate the classical maxentropy $\widetilde{H}_{1 / 2}\left(\left\{n_{x}\right\}\right)$ and the rate $\widetilde{r}\left(\left\{n_{x}\right\}\right)$. For each $m$, in figure 2 we show the average rate $\widetilde{r}$ and its standard deviation experimentally evaluated over 200 different $X$ sequences of $n_{X}$ bits (see Appendix $\mathrm{C}$ for the rate achieved, for each $m$, by a single $X$ sequence of $n_{X}$ bits). The experimental rates can be compared with the 


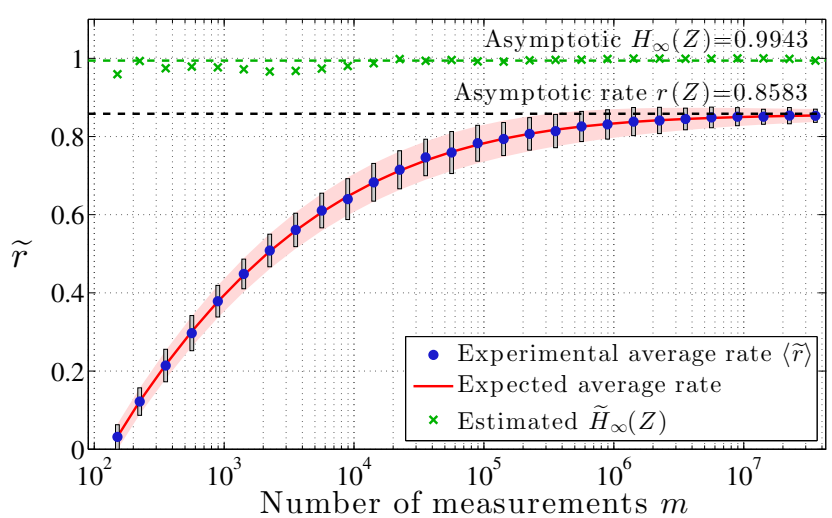

FIG. 2. (Color online) Average experimental rate for the qubit QRNG. Blue circles represent the experimental average rate $\widetilde{r}$ of true random bits per measurement, while the continuous red line is the theoretical prediction with $\rho_{X}=$ $\sum_{x=0}^{1} p_{x}|x\rangle\langle x|$ where $p_{0}=0.9973$ and $p_{1}=0.0027$. Shaded red area represents the theoretical standard deviation of the rate, while gray rectangles show the experimental standard deviation of the rate. Green crosses show the classical minentropy estimated on the $Z$ random variable. The asymptotic limit $H_{\infty}(Z)$ is evaluated on the state $\rho_{Z}=\sum_{z=0}^{1} P_{z}|z\rangle\langle z|$ with $P_{0}=0.5020$ and $P_{1}=0.4980$.

predicted average rate $\langle\widetilde{r}\rangle=\sum_{\left\{n_{x}\right\}} \Pi\left(\left\{n_{x}\right\}\right) \widetilde{r}\left(\left\{n_{x}\right\}\right)$, obtained by averaging $\widetilde{r}\left(\left\{n_{x}\right\}\right)$ over the multinomial distribution $\Pi\left(\left\{n_{x}\right\}\right)=\frac{n_{X} !}{n_{0} ! n_{1} ! \cdots n_{d-1} !} p_{0}^{n_{0}} p_{1}^{n_{1}} \cdots p_{d-1}^{n_{d-1}}$. We also show the classical min-entropy $\widetilde{H}_{\infty}(Z)$ evaluated on a sequence $Z$ with $n_{Z}$ bits. The figure shows a very good agreement between the experimental result and the theoretical prediction. It is worth noticing that at least $m>150$ measurements are necessary to obtain a positive rate $\widetilde{r}$, while with just $m \simeq 10^{6}$ the rate is very close to the asymptotic bound $r(Z)$. The difference between $H_{\infty}(Z)$ and $\widetilde{r}$ corresponds to the possible knowledge that an adversary holding the system $E$ may have. The limit $H_{\infty}(Z)$ is often and erroneously taken as the amount of true randomness used to calibrate the extractor: in this way, even if the output string appears statistically good, possible side information held by Eve is not completely erased. In our experimental analysis, since we are mainly interested in demonstrating the physical principles, we did not use active switches to change between the two bases (we first measured the $Z$ sequence and afterwards the $X$ sequence). For practical applications, however, the QRNG should contain an active switch controlled by the seed $t(m)$.

In figure 3 the results for the ququart QRNG are presented. Also in this case, for each $m$, the average rate $\widetilde{r}$ and its standard deviation are experimentally obtained by 200 different $X$ sequences of $n_{X}(m)$ bits. Again, there is a very good agreement between the experimental results and the theoretical predictions and a positive (average) rate is obtained for $m>70$. As before, for $m \simeq 10^{6}$ the rate is very close to the asymptotic bound $r(Z)$ :

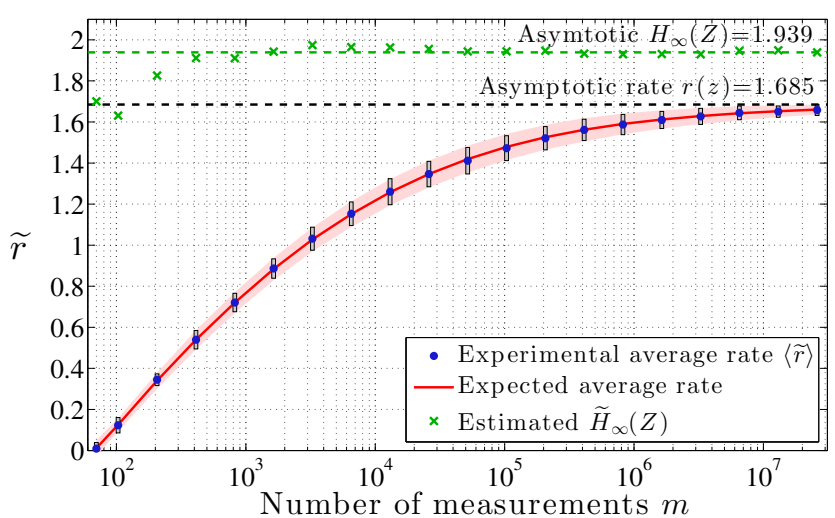

FIG. 3. (Color online) Average experimental rate for the ququart QRNG. See figure 2 for notations. In this case $\rho_{X}=$ $\sum_{x=0}^{3} p_{x}|x\rangle\langle x|$ with $p_{0}=0.9937, p_{1}=0.00359, p_{2}=0.00266$ and $p_{3}=1-p_{0}-p_{1}-p_{2}$ and $\rho_{Z}=\sum_{z=0}^{3} P_{z}|z\rangle\langle z|$ with $P_{0}=0.2527, P_{1}=0.2412, P_{2}=0.2608$ and $P_{3}=0.2453$.

thanks to the larger Hilbert space, we can asymptotically obtain 1.685 bits per measurement, that should be compared with the value 0.8583 achieved with the qubit QRNG. Our method is thus very robust with respect to the increasing of the dimension $d$ of the system.

For the complete proof of our protocol, we performed the extraction on a long random sequence $Z$ and the results are presented in Appendix D.

\section{A. Detailed comparison with Ref. [2]}

Here we give a detailed comparison between our method and the result of Fiorentino et al [2], where the conditional min-entropy of a qubit state is evaluated by measuring its density matrix $\rho=\frac{1}{2}(\mathbb{1}+\vec{r} \cdot \vec{\sigma})$ $\left(\sigma_{i}\right.$ 's are the Pauli matrices and $\vec{r}$ is a three-dimensional vector such that $|\vec{r}| \leq 1$ ). By extracting the random bits by measuring the qubit in the computational basis $\mathbb{Z}=\{|0\rangle,|1\rangle\}$ such that $r_{z}=\langle 0|\rho| 0\rangle-\langle 1|\rho| 1\rangle$, the conditional min-entropy was estimated to be $H_{\min }(Z \mid E)=$ $1-\log _{2}\left(1+\sqrt{1-r_{x}^{2}-r_{y}^{2}}\right)[2]$.

Our method estimates the min-entropy of the $Z$ outcomes by measuring in the $\mathbb{X}=\{| \pm\rangle\}$ basis giving the asymptotic bound of $H_{\min }(Z \mid E) \geq 1-\log _{2}\left(1+\sqrt{1-r_{x}^{2}}\right)$. Our result is a lower bound, since $q-H_{1 / 2}(X)=1-$ $\log _{2}\left[1+\sqrt{1-r_{x}^{2}}\right]$ : the bound is tight when $r_{y}=0$. If the state is pure, the result of [2] allows to achieve the upper limit $H_{\min }(Z \mid E)=H_{\infty}(Z)$. The advantage of our approach resides in the fact that it is not necessary to measure the full density matrix but only measurements on two mutually-unbiased basis. Indeed, in order to evaluate the density matrix, it is necessary to measure the system also in the $\mathbb{X}$ and $\mathbb{Y}=\left\{\frac{1}{\sqrt{2}}(|0\rangle \pm i|1\rangle)\right\}$ basis beside the basis chosen to obtain the random sequence. Also in the case of [2], a random seed is needed to switch be- 
tween the tomography bases and the random sequence basis. As a final consideration, the result of Fiorentino et al. applies only to qubit systems, while our result can be applied to a general qudit systems, as we have demonstrated by analyzing the ququart QRNG.

We now give a detailed comparison for finite $m$ : let's consider the following parameters $r_{z}=0.9947 \pm 0.001$ and $r_{x}=0.004 \pm 0.002$ corresponding to the experimental measured parameter of our qubit QRNG. Since the norm of the vector $\vec{r}$ cannot be greater that 1 , it implies that $\left|r_{y}\right| \leq \sqrt{1-r_{z}^{2}-r_{x}^{2}} \leq 0.1027$ corresponding to a purity greater that $\mathcal{P}_{\text {min }}=0.9947$. We recall that purity of the state $\rho$ is defined as $\mathcal{P}=\operatorname{Tr}\left[\rho^{2}\right]=\frac{1+r_{x}^{2}+r_{y}^{2}+r_{z}^{2}}{2}$. The measurement in the $Y$ basis will allow to determine the $r_{y}$ parameter.

We performed the detailed comparison, in the finite $m$ case ( $m$ is the total number of measurements), between our method and Ref. 22. To obtain a fair comparison we set $n_{X}^{*}=n_{Y}^{*}=\lceil\sqrt{m} / 2\rceil$ as the number of measurements in the $X$ and $Y$ basis respectively for the tomographic method of [2]. Then the number of measurements in the $Z$ basis is given by $n_{Z}^{*}=m-2\lceil\sqrt{m} / 2\rceil$. From such measurements the $r_{x}$ and $r_{y}$ parameters are estimated as (we used Bayesian estimators):

$$
r_{x}=\frac{n_{0 x}-n_{1 x}}{n_{0 x}+n_{1 x}+2} \quad r_{y}=\frac{n_{0 y}-n_{1 y}}{n_{0 y}+n_{1 y}+2}
$$

To randomly choose the $X$ and $Y$ measurements over the total number of measurements $m$ we need a number of bits given by $t^{*}(m)=2\left\lceil\log _{2} \frac{m !}{\left(2 n_{X}^{*}\right) !\left(m-2 n_{X}^{*}\right) !}\right\rceil$.

In Fig. 5 we show the comparison between the two rates in case of perfect pure state $\mathcal{P}=1$ and in the case of $r_{y}=0$, corresponding to $\mathcal{P}=0.995$ : the figure show that our results are slightly outperformed by the tomographic extractor only for high purity states $\mathcal{P}>0.995$ and in the large $m$ regime $\left(m>10^{5}\right)$. A maximum of $15 \%$ improvement with respect to the results shown in Fig. 2 is expected if the generated state is pure $\mathcal{P}=1$ and $N>10^{8}$. However, to obtain such limited advantage, a complication in the scheme, namely the measurement in the $Y$ basis, is required.

\section{CONCLUSIONS}

We provided a bound, given by equation (5), to directly compute the conditional min-entropy $H_{\min }(Z \mid E)$ of the random variable $Z$, by using the classical random variable $X$. The variables $Z$ and $X$ are obtained by measuring the system in two mutually unbiased bases. $H_{\min }(Z \mid E)$ represents the amount of true randomness that can be extracted from $Z$. No assumption is made on the source and/or the dimension of Hilbert space. Our result is based on the fact the measurement device is trusted: we assumed that the measurement system (waveplates and PBSs) works properly and the detector efficiency is not dependent on the input state or on an external control.

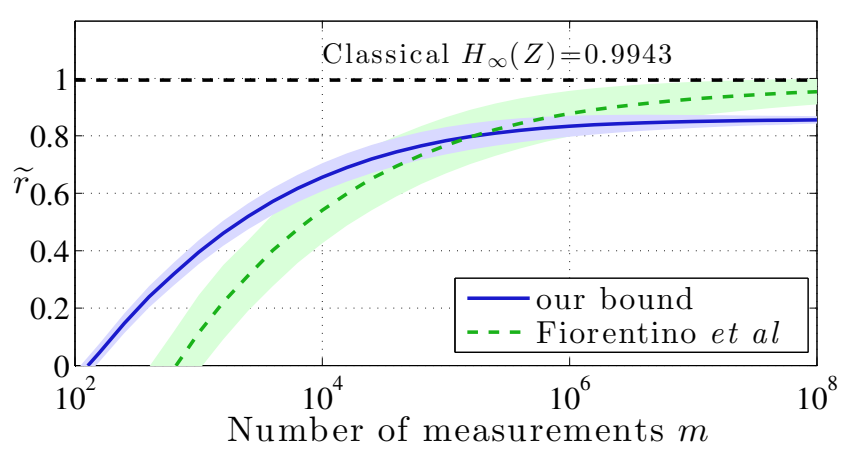

FIG. 4. (Color online) Comparison between the rate achievable by our bound (continuous blu line) and the rate achievable with the min-entropy estimation of Ref. 2] (dotted green line) in the case of perfect pure state with purity $\mathcal{P}=1$.

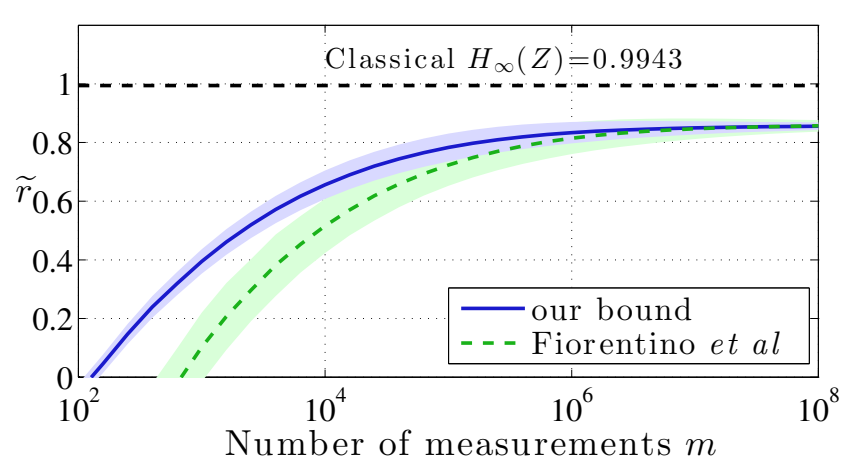

FIG. 5. (Color online) Comparison between the rate achievable by our bound (continuous blu line) and the rate achievable with the min-entropy estimation of Ref. 2] (dotted green line) in the case of slightly mixed state with purity $\mathcal{P}=0.995$.

In order that detection system is only sensitive to a well known and characterized finite dimensional subspace of the total Hilbert space, photon number resolving detectors or the squashing model of QKD [28, 29] can be implemented. It is important to stress that if the source does not generate a perfect pure state (and this always happens in experimental realizations), the randomness extracted by standard methods, namely by measuring the system in a single basis, is not a true randomness: an eavesdropper can have (partial or full) information about the generated random bits. We have also tested our bound with a qubit and a ququart QRNG with good agreement between theory and experiment.

Our method can be extended by taking into account possible imperfections in the measurement device, as illustrated in 16. We believe that our method can be very useful for the extraction of true randomness and can be applied in the framework of practical high-speed QRNG [6, 8, since it guarantees protection against quantum side information without the need of complex Bell violation experiment. 


\section{ACKNOWLEDGMENTS}

We would like to thank Alberto Dall'Arche of the University of Padova for his support on the setup preparation. Our work was supported by the Strategic-ResearchProject QUINTET of the Department of Information Engineering, University of Padova and the StrategicResearch-Project QUANTUMFUTURE (STPD08ZXSJ) of the University of Padova.

\section{Appendix A: Min and Max-entropy}

We here briefly review the definition of conditional min- and max- entropies introduced in [18. The conditional min-entropy of a bipartite quantum state $\rho_{A E}$ is defined as:

$$
H_{\min }(A \mid E)_{\rho_{A E}}=\max _{\sigma_{B}} \sup \left\{\lambda \in \mathbb{R} \mid \frac{\mathbb{1}_{A} \otimes \sigma_{E}}{2^{\lambda}} \geq \rho_{A E}\right\},
$$

where $\sigma_{E}$ is a normalized positive state.

The conditional max-entropy is the dual of the minentropy. In fact, by using a purification $\rho_{A B C}$ of $\rho_{A B}$, the max-entropy is defined by

$$
H_{\max }(A \mid B)_{\rho_{A B}}=-H_{\min }(A \mid C)_{\rho_{A C}},
$$

where $\rho_{A B}=\operatorname{Tr}_{C}\left[\rho_{A B C}\right]$ and $\rho_{A C}=\operatorname{Tr}_{B}\left[\rho_{A B C}\right]$. We here recall that the purification of a state $\rho_{A B}$ is a pure state $\rho_{A B C}$ in the extended Hilbert space $A \otimes B \otimes C$, such that $\operatorname{Tr}_{C}\left[\rho_{A B C}\right]=\rho_{A B}$.

For the QRNG we need to evaluate the max-entropy for the state $\rho_{X} \equiv \sum_{x=0}^{d-1} p_{x}|x\rangle\langle x|$, where the space $B$ is a trivial space. By definition A2 we have:

$$
H_{\max }(X)_{\rho_{X}}=-H_{\min }(A \mid C)_{\rho_{A C}}
$$

with $\rho_{A C}$ a purification of $\rho_{X}$. A possible purification is given by

$$
\rho_{A C}=|\Psi\rangle_{A C}\langle\Psi|, \quad| \Psi\rangle_{A C}=\sum_{x=0}^{d-1} \sqrt{p_{x}}|x\rangle_{A} \otimes\left|v_{x}\right\rangle_{C}
$$

with $\left\{\left|v_{x}\right\rangle\right\}$ on orthonormal basis on the space $C$ with dimension $d$. By A1 we have

$$
\begin{aligned}
H_{\max }(X)_{\rho_{X}} & =-H_{\min }(A \mid C)_{\rho_{A C}} \\
& \left.=-\max _{\sigma_{B}} \sup \left\{\lambda \in \mathbb{R}\left|\frac{\mathbb{1}_{A} \otimes \sigma_{C}}{2^{\lambda}} \geq\right| \Psi\right\rangle\langle\Psi|\right\},
\end{aligned}
$$

The state $\sigma_{C}$ that maximize min-entropy definition is $\sigma_{C}=\mathbb{1} / d$. The maximum $\lambda$ such that $\mathbb{1}_{A} \otimes \mathbb{1}_{C} \geq$ $d 2^{\lambda}|\Psi\rangle\langle\Psi|$ is $\lambda=-\log _{2}\left[\sum_{x}\left(\sqrt{p_{x}}\right)\right]^{2}$, such that

$$
H_{\max }(X)_{\rho_{X}}=\log _{2}\left[\sum_{x} \sqrt{p_{x}}\right]^{2}=2 \log _{2} \sum_{x} \sqrt{p_{x}}=H_{1 / 2}(X)
$$

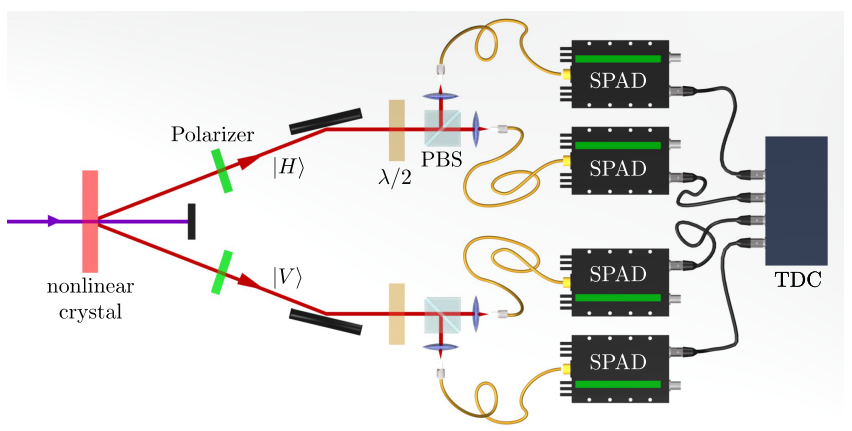

FIG. 6. (Color online) Scheme of the experimental setup generating the SPDC photons. $\mathrm{TDC}=$ Time to digital converter; $\mathrm{SPAD}=$ single-photon avalanche diode $\mathrm{PBS}=$ polarizing beam splitter, $\lambda / 2=$ waveplates.

\section{Appendix B: Photon source}

Photons used in experimental demonstration of the method were generated by spontaneous parametric down conversion (SPDC), as illustrated in figure 6. A femtosecond pulsed laser $(76 \mathrm{MHz}$ repetition rate) at $405 \mathrm{~nm}$ shines a nonlinear crystal where pairs of photons are probabilistically emitted over two correlated directions. Two polarizers select the $|H V\rangle$ pair, with $|H\rangle$ and $|V\rangle$ the horizontal and vertical polarized photon respectively. Half waveplates $\lambda / 2$ allow to change between the $\mathbb{Z}$ and $\mathbb{X}$ basis. For the single qubit QRNG, the $|H\rangle$ photon is used as trigger: its detection heralds the presence of the $|V\rangle$ photon. Single photon detectors (SPAD) deliver signals to a time-to-digital converter (TDC). Concerning the rate of raw bits extraction, the source has a coincidence rate of $12 \mathrm{kHz}$ : we would like to point out that we are not interested in the speed of the generator, but on the demonstration of the method here presented. However, it is worth noticing that sources producing photon pairs at the rate of few $\mathrm{MHz}$ are currently available [30, 31.

\section{Appendix C: Analysis of the random bit generation rate}

In this section we show the experimental rate obtained with a single control $X$ sequence, while in the main text we showed the average value obtained with 200 sequences. We report the rate achieved with the qubit QRNG. We here recall that, given $m$ measurement on the state $\rho_{A}$, we obtained two classical $X$ and $Z$ sequences with $n_{X}$ and $n_{Z}$ bits respectively, whose lengths are respectively given by $n_{X}=\left\lceil\sqrt{m}\right.$ and $n_{Z}=m-n_{X}$. The state of the system $A$ after the measurement is given by $\rho_{Z}=\sum_{z=0}^{1} P_{z}|z\rangle\langle z|$ or $\rho_{X}=\sum_{x=0}^{1} p_{x}|x\rangle\langle x|$, depending on the used POVM.

Given $m$, we would like to evaluate the "single shot" 


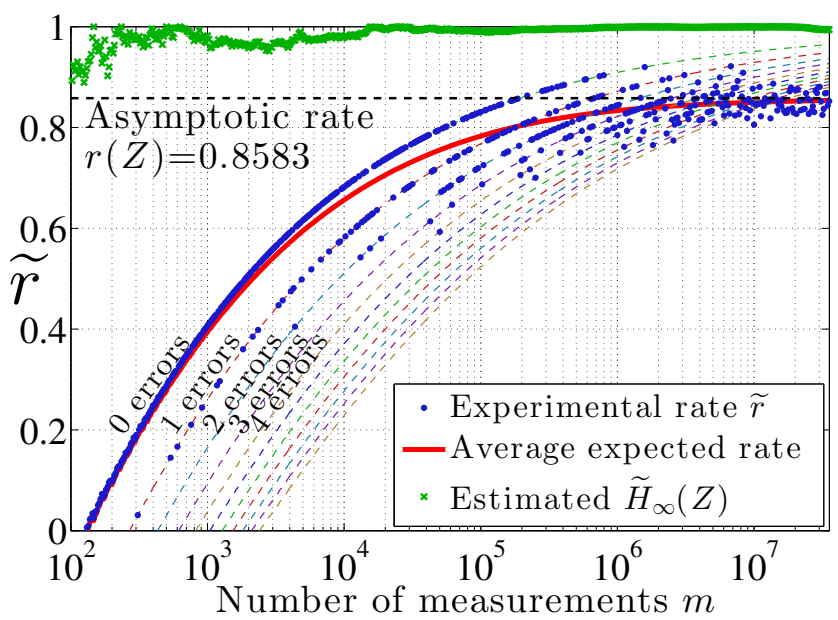

FIG. 7. (Color online) Experimental rate for the qubit RNG. Blu circles represents the experimental rate $\widetilde{r}$ of true random bits per measurement, while continuous red line represent the theoretical average prediction with $\rho_{X}=\sum_{x=0}^{1} p_{x}|x\rangle\langle x|$ where $p_{0}=0.9973$ and $p_{1}=0.0027$. Dashed lines represent the rate achieved with different number of "errors" in the $X$ sequence. Green crosses show the classical min-entropy estimated on the $Z$ random variable obtained from the state $\rho_{Z}=\sum_{z=0}^{1} P_{z}|z\rangle\langle z|$ with $P_{0}=0.5020$ and $P_{1}=0.4980$.

rate $\widetilde{r}$ given by:

$$
\widetilde{r}\left(n_{0}, n_{1}, m\right)=\left(m-n_{X}\right)\left(1-\widetilde{H}_{1 / 2}\left(n_{0}, n_{1}\right)\right)-t(m),
$$

with $n_{0}$ and $n_{1}$ the number of 0 's and 1's in the $X$ sequence.

For the single qubit QRNG, since $n_{0}+n_{1}=n_{X}$, the single shot rate is function of only $m$ and $n_{1}$ :

$$
\begin{aligned}
\widetilde{r}\left(n_{1}, m\right)= & \left(m-n_{X}\right)\left\{1-2 \log _{2}\left[\frac{\Gamma\left(n_{X}+2\right)}{\Gamma\left(n_{X}+\frac{5}{2}\right)}\right]\right. \\
& \left.-2 \log _{2}\left[\frac{\Gamma\left(n_{X}-n_{1}+\frac{3}{2}\right)}{\Gamma\left(n_{X}-n_{1}+1\right)}+\frac{\Gamma\left(n_{1}+\frac{3}{2}\right)}{\Gamma\left(n_{1}+1\right)}\right]\right\} \\
& -\left\lceil\log _{2}\left(\begin{array}{c}
m \\
n_{X}
\end{array}\right)\right\rceil .
\end{aligned}
$$

For different values of $m$ we show in figure 7 the achieved rate: each point represents the rate $\widetilde{r}$ evaluated over a single $X$ sequence of $n_{X}$ bits obtained by the measurement in the $\mathbb{X}$ POVM. Each sequence is taken from a sample with the following property:

$$
\rho_{X}=\sum_{x=0}^{1} p_{x}|x\rangle\langle x| \quad \text { with } \quad p_{0}=0.9973, p_{1}=0.0027 .
$$

For perfect state preparation we would like to have $p_{0}=1$ and $p_{1}=0$ : by this reason, the number of 1 in the $X$ sequence are defined as the "number of errors" in the sequence. The "errors" can be caused by the presence of the eavesdropper, or by imperfections in the preparation
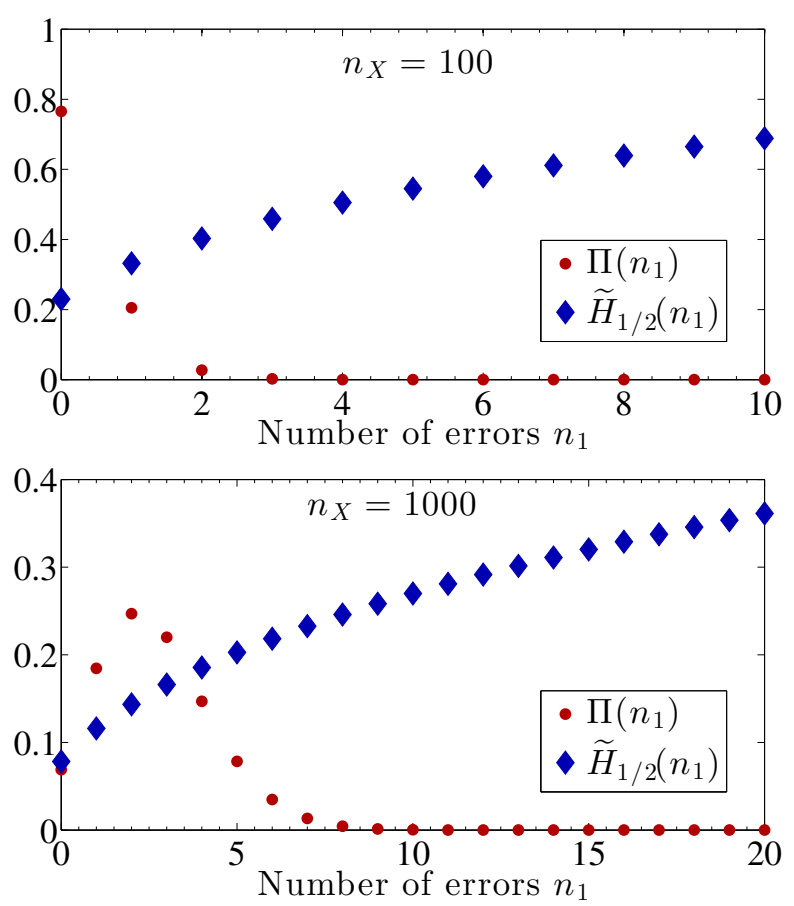

FIG. 8. (Color online) Extimated max-entropy $\widetilde{H}_{1 / 2}(X)$ and error probability $\Pi\left(n_{1}\right)$. Due to the low value of $p_{1}=0.0027$, the $\Pi\left(n_{1}\right)$ is peaked around the low values of $n_{1}$.

devices. Since $p_{1}$ is very low, in Figure 7 it is possible to see that, for $m<10^{3}$, few sequences have 1 errors and the most have 0 errors. By increasing $m$, the number of errors increases to follow the prediction $n_{1} \sim p_{1} n_{X}$. For low $m$, the possible rates are "quantized", since the rate is evaluated on integer values $n_{0}$ and $n_{1}$. In figure 8 we show estimated max-entropy $\widetilde{H}_{1 / 2}(X)$ in function of the number of errors for the $n_{X}=100$ and $n_{X}=1000$ case. We also report the probability of obtaining $n_{1}$ errors, given by $\Pi\left(n_{1}\right)=\left(\begin{array}{c}n_{X} \\ n_{1}\end{array}\right) p_{0}^{n_{0}} p_{1}^{n_{1}}$. The figure shows that $\widetilde{H}_{1 / 2}(X)$ has discrete values corresponding to different values of $n_{1}$.

\section{Appendix D: Tests on the extracted random numbers}

As a quantitative example for the complete proof of our method, we performed the extraction on a long random sequence $Z$. For the qubit case we use a random sequence $Z$ of length $n_{Z}=35.6 \cdot 10^{6}$ and a control sequence $X$ of length $n_{X}=5967$, requiring a seed length $t(m)=83443$. The estimated lower bound for the minentropy is $1-H_{1 / 2}(X) \simeq 0.8437$ giving an output random sequence $Y$ of $b_{\mathrm{sec}} \simeq 29.951 \cdot 10^{6} \mathrm{bits}$. For the qudit case, we have $n_{Z}=25.770 \cdot 10^{6}$ and $n_{X}=5100$ with a seed length $t(m)=70163$. The estimated lower bound for the min-entropy is 1.690 , giving $b_{\mathrm{sec}} \simeq 43.886 \cdot 10^{6}$ true 


\begin{tabular}{|c|c|c|c|c|c|c|}
\hline Suite & Test & P-Value & Suite & Test & \multicolumn{2}{|c|}{ P-Value } \\
\hline \multirow{25}{*}{$\begin{array}{l}R \\
\text { a } \\
b \\
b \\
i \\
t\end{array}$} & MultinomialBitsOver & 0.94 & \multirow{9}{*}{$\begin{array}{l}\text { A } \\
\text { I } \\
\text { p } \\
\text { h } \\
\text { a } \\
\text { b } \\
\text { i } \\
\text { t }\end{array}$} & MultinomialBitsOver $(\mathrm{L}=2)$ & \multicolumn{2}{|c|}{0.72} \\
\hline & ClosePairsBitMatch (t=2) & 0.10 & & MultinomialBitsOver $(\mathrm{L}=4)$ & \multicolumn{2}{|c|}{0.38} \\
\hline & ClosePairsBitMatch $(\mathrm{t}=\mathbf{4})$ & 0.10 & & MultinomialBitsOver $(\mathrm{L}=8)$ & \multicolumn{2}{|c|}{0.81} \\
\hline & AppearanceSpacings & 0.76 & & MultinomialBits Over $(L=16)$ & \multicolumn{2}{|c|}{0.38} \\
\hline & LinearComp & 0.23 & & HammingIndep $(L=16)$ & \multicolumn{2}{|c|}{0.02} \\
\hline & LempelZiv & 0.32 & & HammingIndep $(L=32)$ & \multicolumn{2}{|c|}{0.64} \\
\hline & Fourier1 & 0.60 & & HammingCorr $(L=32)$ & \multicolumn{2}{|c|}{0.72} \\
\hline & Fourier3 & 0.53 & & RandomWalk1 ( $L=64)$ & \multicolumn{2}{|c|}{0.04} \\
\hline & LongestHeadRun & 0.59 & & RandomWalk1 $(\mathrm{L}=320)$ & \multicolumn{2}{|c|}{0.03} \\
\hline & PeriodsInStrings & 0.950 & & Test & P-Val. & Pass Ratio \\
\hline & HammingWeight $(\mathrm{L}=32)$ & 0.24 & & Frequency & 0.066882 & $149 / 150$ \\
\hline & HammingCorr $(\mathrm{L}=32)$ & 0.72 & & BlockFrequency & 0.299251 & $148 / 150$ \\
\hline & HammingCorr $(L=64)$ & 0.65 & & CumulativeSums & 0.431143 & $149 / 150$ \\
\hline & HammingCorr $(\mathrm{L}=128)$ & 0.54 & & CumulativeSums & 0.588652 & $148 / 150$ \\
\hline & HammingIndep $(L=16)$ & 0.02 & & Runs & 0.671779 & $150 / 150$ \\
\hline & HammingIndep $(L=32)$ & 0.64 & \multirow{3}{*}{$\begin{array}{l}8 \\
0 \\
0\end{array}$} & LongestRun & 0.911413 & $149 / 150$ \\
\hline & HammingIndep $(L=64)$ & 0.89 & & Rank & 0.132132 & 149/150 \\
\hline & AutoCor with a $\operatorname{lag} d=1$. & 0.79 & & FFT & 0.142602 & $148 / 150$ \\
\hline & AutoCor with a lag $d=2$. & 0.86 & \multirow{4}{*}{$\overline{2}$} & NonOverlappingTemplate & \multicolumn{2}{|c|}{$148 / 148$ (subtests) } \\
\hline & Run & 0.20 & & OverlappingTemplate & 0.056546 & $150 / 150$ \\
\hline & MatrixRank $(32 \times 32)$ & 0.56 & & ApproximateEntropy & 0.520767 & $148 / 150$ \\
\hline & RandomWalk1 ( $L=128)$ & 0.12 & & RandomExcursion & \multicolumn{2}{|c|}{$26 / 26$ (subtests) } \\
\hline & RandomWalk1 (L = 1024) & 0.12 & & Serial $(M=16)$ & 0.712961 & 149/150 \\
\hline & RandomWalk1 (L = 10016) & 0.01 & & Serial $(M=16)$ & 0.236810 & $150 / 150$ \\
\hline & & & & LinearComplexity & 0.092784 & $148 / 150$ \\
\hline
\end{tabular}

\begin{tabular}{|c|c|c|c|c|c|c|}
\hline Suite & Test & P-Value & Suite & Test & \multicolumn{2}{|c|}{ P-Value } \\
\hline & MultinomialBitsOver & 0.27 & \multirow{9}{*}{$\begin{array}{l}\text { A } \\
\text { I } \\
\text { p } \\
\text { h } \\
\text { a } \\
\text { b } \\
\text { i } \\
\text { t }\end{array}$} & MultinomialBitsOver $(\mathrm{L}=2)$ & \multicolumn{2}{|c|}{0.19} \\
\hline & ClosePairsBitMatch (t=2) & 0.20 & & MultinomialBitsOver $(\mathrm{L}=4)$ & \multicolumn{2}{|c|}{0.23} \\
\hline & ClosePairsBitMatch $(t=4)$ & 0.58 & & MultinomialBitsOver $(\mathrm{L}=8)$ & \multicolumn{2}{|c|}{0.64} \\
\hline & AppearanceSpacings & 0.67 & & MultinomialBitsOver $(\mathrm{L}=16)$ & \multicolumn{2}{|c|}{0.94} \\
\hline & LinearComp & 0.19 & & Hamminglndep $(L=16)$ & \multicolumn{2}{|c|}{0.73} \\
\hline & LempelZiv & 0.84 & & Hammingindep $(L=32)$ & \multicolumn{2}{|c|}{0.73} \\
\hline & Fourier1 & 0.50 & & HammingCorr $(L=32)$ & \multicolumn{2}{|c|}{0.52} \\
\hline & Fourier3 & 0.38 & & RandomWalk1 $(\mathrm{L}=64)$ & \multicolumn{2}{|c|}{0.29} \\
\hline & LongestHeadRun & 0.04 & & RandomWalk1 $(\mathrm{L}=320)$ & \multicolumn{2}{|c|}{0.15} \\
\hline & PeriodsInStrings & 0.37 & \multicolumn{2}{|r|}{ Test } & P-Val. & Pass Ratio \\
\hline $\mathbf{R}$ & HammingWeight $(\mathrm{L}=32)$ & 0.51 & & Frequency & 0.759756 & $97 / 100$ \\
\hline $\mathbf{a}$ & HammingCorr $(L=32)$ & 0.52 & & BlockFrequency & 0.964295 & $100 / 100$ \\
\hline b & HammingCorr $(L=64)$ & 0.70 & & CumulativeSums & 0.096578 & $97 / 100$ \\
\hline b & HammingCorr $(\mathrm{L}=128)$ & 0.13 & $\mathbf{s}$ & CumulativeSums & 0.911413 & $97 / 100$ \\
\hline $\mathbf{i}$ & HammingIndep $(L=16)$ & 0.73 & $\mathbf{P}$ & Runs & 0.779188 & $98 / 100$ \\
\hline \multirow[t]{11}{*}{$\mathrm{t}$} & HammingIndep ( $L=32)$ & 0.73 & - & LongestRun & 0.494392 & $99 / 100$ \\
\hline & HammingIndep $(L=64)$ & 0.71 & 8 & Rank & 0.011791 & $100 / 100$ \\
\hline & AutoCor with a lag $\mathrm{d}=1$. & 0.36 & 0 & FFT & 0.657933 & $100 / 100$ \\
\hline & AutoCor with a lag $d=2$. & 0.26 & 0 & NonOverlappingTemplate & \multicolumn{2}{|c|}{ 148/148 (subtests) } \\
\hline & Run & 0.02 & - & OverlappingTemplate & 0.816537 & $99 / 100$ \\
\hline & MatrixRank $(32 \times 32)$ & 0.39 & 2 & Universal & 0.289667 & $99 / 100$ \\
\hline & MatrixRank $(320 \times 320)$ & 0.84 & 2 & ApproximateEntropy & 0.867692 & $100 / 100$ \\
\hline & RandomWalk1 ( $L=128)$ & 0.21 & & RandomExcursion & \multicolumn{2}{|c|}{$26 / 26$ (subtests) } \\
\hline & RandomWalk1 $(\mathrm{L}=1024)$ & 0.51 & & Serial $(M=16)$ & 0.798139 & $98 / 100$ \\
\hline & RandomWalk1 $(\mathrm{L}=10016)$ & 0.24 & & Serial $(M=16)$ & 0.514124 & $99 / 100$ \\
\hline & & & & LinearComplexity & 0.401199 & $100 / 100$ \\
\hline
\end{tabular}

TABLE I. (left) Summary of the results of selected tests of batteries particularly effective in detecting defects in TRNG. The Alphabit and Rabbit batteries belong to the TESTU01: critical results are if $\mathcal{P}$-val $\leq 10^{-3}$ or $\mathcal{P}$-val $\geq 0.990$. For tests which give more than a p-values, the smallest is reported. For NIST SP-800-22 suite, the file was partitioned in sub-strings 200000 bits long for a total of 150 strings: this length was chosen in order to obtain a sample sizes enough large such that it is likely to fail the tests in case of poor randomness with a significance level of $\alpha=0.01$; a test is failed if more than 6 strings fail it. In addition, a test is passed if the a chi-square test on the distribution of p-values, gives it self a p-value $\mathcal{P}$-val $\geq 10^{-5}$. (right) Summary of the results of selected tests of batteries particularly effective in detecting defects in TRNG. The Alphabit and Rabbit batteries belong to the TESTU01: critical results are if $\mathcal{P}$-val $\leq 10^{-3}$ or $\mathcal{P}$-val $\geq 0.990$. For tests which give more than a p-values, the smallest is reported. For NIST SP-800-22 suite, the file was partitioned in sub-strings 400000 bits long for a total of 100 strings: this length was chosen in order to obtain a sample sizes enough large such that it is likely to fail the tests in case of poor randomness with a significance level of $\alpha=0.01$; a test is failed if more than 4 strings fail it. In addition, a test is passed if the a chi-square test on the distribution of p-values, gives it self a p-value $\mathcal{P}$-val $\geq 10^{-5}$.

random bits. In both case, the initial $Z$ strings are fed to an extractor by two-universal hashing [16, 32, to obtain the $Y$ strings. As we now will shown, the obtained bits pass successfully the most stringent tests [33] for the assessment of i.i.d. hypothesis for random bits.

At present time, the TEST-U01 34] is the most stringent and comprehensive suite of tests; among all, we chose a pair sub-batteries, Rabbit and Alphabit respectively, specifically designed to tests RNGs. The SP-80022 [33] is developed by the NIST and it is the most applied battery for RNG evaluation.

The output of a test on a bit string is another random variable with a given distribution of probability, the so-called test statistic. Hence, the $\mathcal{P}$-value, namely the probability of getting an equal or worse test statistic, holding true the i.i.d. hypothesis, are computed. If the $\mathcal{P}$-values are smaller than some a priori defined critical values the tests are considered failed: these limits are usually chosen as $\mathcal{P}$-value $<0.01$ and $\mathcal{P}$-value $<0.001$, corresponding to a confidence level of $99 \%$ and $99.9 \%$ respectively. Otherwise, whenever one obtains $\mathcal{P}$-values equal or greater than these limits, the i.i.d. hypothesis for the tested string is assessed.

In Table I we report the results applied on the secure bits extracted by measuring a qubit and a ququart respectively. All the tests are passed.
[1] T. Jennewein, U. Achleitner, G. Weihs, H. Weinfurter, and A. Zeilinger, Review of Scientific Instruments 71, 1675 (2000).

[2] M. Fiorentino, C. Santori, S. M. Spillane, R. G. Beausoleil, and W. J. Munro, Physical Review A 75, 032334 (2007).

[3] W. Wei and H. Guo, Optics Letters 34, 1876 (2009).

[4] K. Svozil, Physical Review A 79, 054306 (2009).

[5] M. Fürst, H. Weier, S. Nauerth, D. G. Marangon, C. Kurtsiefer, and H. Weinfurter, Optics Express 18, 13029 (2010).

[6] M. Jofre, M. Curty, F. Steinlechner, G. Anzolin, J. P.
Torres, M. W. Mitchell, and V. Pruneri, Optics Express 19, 20665 (2011).

[7] R. Gallego, L. Masanes, G. De La Torre, C. Dhara, L. Aolita, and A. Acín, Nature Communications 4, 2654 (2013).

[8] C. Abellán, W. Amaya, M. Jofre, M. Curty, A. Acín, J. Capmany, V. Pruneri, and M. W. Mitchell, Optics Express 22, 1645 (2014).

[9] S. Pironio, A. Acín, S. Massar, A. B. de la Giroday, D. N. Matsukevich, P. Maunz, S. Olmschenk, D. Hayes, L. Luo, T. A. Manning, and C. Monroe, Nature (London) 464, 1021 (2010). 
[10] C. Dhara, G. Prettico, and A. Acín, Physical Review A 88, 052116 (2013).

[11] R. Colbeck, Quantum And Relativistic Protocols For Secure Multi-Party Computation, Ph.D. thesis (2006), arXiv:0911.3814v2].

[12] R. Colbeck and A. Kent, Journal of Physics A: Mathematical and Theoretical 44, 095305 (2011).

[13] U. Vazirani and T. Vidick, Philosophical transactions. Series A, Mathematical, physical, and engineering sciences 370, 3432 (2012).

[14] S. Pironio and S. Massar, Physical Review A 87, 012336 (2013).

[15] R. Colbeck and R. Renner, Nature Physics 8, 450 (2012).

[16] D. Frauchiger, R. Renner, and M. Troyer, arXiv:1311.4547. (2013).

[17] A. De, C. Portmann, T. Vidick, and R. Renner, SIAM Journal on Computing 41, 915 (2012).

[18] R. Konig, R. Renner, and C. Schaffner, IEEE Transactions on Information Theory 55, 4337 (2009).

[19] Y. Zhao, C.-H. Fung, B. Qi, C. Chen, and H.-K. Lo, Physical Review A 78, 042333 (2008).

[20] L. Lydersen, C. Wiechers, C. Wittmann, D. Elser, J. Skaar, and V. Makarov, Nature Photonics 4, 686 (2010).

[21] F. Xu, B. Qi, and H.-K. Lo, New Journal of Physics 12, 113026 (2010).

[22] L. Lydersen, V. Makarov, and J. Skaar, Phys. Rev. A 83, 032306 (2011).
[23] M. Tomamichel and R. Renner, Physical Review Letters 106, 110506 (2011).

[24] J. Renes and J.-C. Boileau, Physical Review Letters 103, 020402 (2009).

[25] M. Berta, M. Christandl, R. Colbeck, J. M. Renes, and R. Renner, Nature Physics 6, 659 (2010).

[26] H. Maassen and J. B. M. Uffink, Phys. Rev. Lett. 60, 1103 (1988).

[27] D. Holste, I. Groß e, and H. Herzel, Journal of Physics A: Mathematical and General 31, 2551 (1998).

[28] N. Beaudry, T. Moroder, and N. Lütkenhaus, Physical Review Letters 101, 093601 (2008).

[29] O. Gittsovich, N. J. Beaudry, V. Narasimhachar, R. R. Alvarez, T. Moroder, and N. Lütkenhaus, Physical Review A 89, 012325 (2014).

[30] F. Steinlechner, P. Trojek, M. Jofre, H. Weier, D. Perez, T. Jennewein, R. Ursin, J. Rarity, M. W. Mitchell, J. P. Torres, H. Weinfurter, and V. Pruneri, Optics Express 20, 9640 (2012).

[31] F. Steinlechner, S. Ramelow, M. Jofre, M. Gilaberte, T. Jennewein, J. P. Torres, M. W. Mitchell, and V. Pruneri, Optics Express 21, 11943 (2013).

[32] L. Trevisan, Journal of the ACM 48, 860 (2001).

[33] E. Rukhin, Andrew and Soto, Juan and Nechvatal, James and Smid, M and Barker, NIST Special Publication 80022 Revision 1a (2010).

[34] P. L'Ecuyer and R. Simard, ACM Transactions on Mathematical Software 33, 22 (2007). 